\title{
Revealing the 3-dimensional shape of atom probe tips by atomic force microscopy
}

C. Fleischmann ${ }^{1 *}$, K. Paredis ${ }^{1}$, D. Melkonyan ${ }^{1,2}$, W. Vandervorst ${ }^{1,2}$

${ }^{1}$ imec, Kapeldreef 75, 3001 Heverlee, Belgium

2 Instituut voor Kern- en Stralingsfysica, KU Leuven, Celestijnenlaan 200D, 3001 Leuven, Belgium

(*) Corresponding author

\begin{abstract}
For the very first time, atomic force microscopy is used to determine quantitatively the 3dimensional shape of an atom probe tip, which is key towards improved accuracy and understanding of artefacts in atom probe tomography. We have successfully measured by atomic force microscopy the apex and shank region of 3 different atom probe tips, of which two show (severe) deviations from a hemisphere due to either non-uniform laser light absorption or the presence of two different materials. Clearly, our method which overcomes the challenge of aligning two very sharp tips on top of each other, offers new pathways to study physical mechanisms in (laser-assisted) atom probe. It represents an important step towards improved data-driven reconstruction algorithms as the image formation in atom probe tomography is based on the intricate link between the tip shape (down to the atomic level), the electric field distribution and the ions' flight path towards the detector. Further on, present reconstruction algorithms solely account for a hemispherical tip shape, which does not hold true for most applications and results in complex artefacts. Therefore our method is an attractive novel approach to assess the 3D tip shape.
\end{abstract}

\section{Keywords}

Non-hemispherical emitter shape, tip radius, tip shape evolution, atomic force microscopy, AFM, TEM, APT, reconstruction artefacts, tip convolution 


\section{Introduction}

In an atom probe microscope, the sample itself is the most critical optical component, hence its shape is crucial to the performance of the technique [1]. Relying on the principle of (laseror voltage-assisted) controlled field ionization and evaporation of surface atoms, the end shape of the conically shaped sample (i.e. tip) should not exceed $100 \mathrm{~nm}$ in diameter to achieve the necessary field enhancement of several tens of $\mathrm{V} / \mathrm{nm}$ [2]. Furthermore, the (local) shape of that tip - down to the atomic level - such as its shank angle, apex curvature, atomic terraces and its local dielectric properties etc. directly define the electric field distribution in far- and near-field $[1,3,4,5,6]$. This, in turn, dictates the ion trajectories, i.e. the ion's projection onto the position-sensitive detector and hence the image formation. Effects such as local magnification variations (and apparent density variations) arise from local differences in the tip curvature as these cause local (de)focusing of the ion trajectories. Their existence violates the basic assumption (i.e. constant magnification over the tip surface) of the standard data reconstruction algorithms whereby the ions' impact position is back-projected onto a hemispherical specimen end-form $[7,8,9]$. As a result, atoms are misplaced in $x, y$ plane as well as $z$ direction in the reconstructed volume, therewith reducing the spatial accuracy and yielding erroneous dimensions and/or artefacts like local density variations and geometrical errors $[4,5,10]$. If, in some instances, ion trajectories cross, also compositional accuracy might be degraded [4] and spatial information can no longer be retrieved [11]. Notorious examples are magnification variations between different materials, at grain boundaries, disrupted/defective interfaces, etc. These occur in particular in heterogeneous systems [12, 13] whereby within the semiconductor field embedded FinFETs represent a typical case [4, 14]. Even for bulk materials (e.g. semiconductors, steels, oxides) the deviation from an hemispherical tip shape due to green $[15,16,17]$ or UV $[18,19]$ laser light exposure is widely acknowledged.

Obviously the way forward towards improved accuracy in APT, lies in a more refined reconstruction approach whereby the detailed tip shape (and the resulting field distribution) is effectively treated as an ion optical component determining the ion trajectories and their impact position on the detector [3]. As a logical consequence, detailed knowledge of the tip shape, preferably at all length scales, before, after and prospectively during atom probe analysis, then becomes key to properly account for its role and to improve accuracy and precision in the atom positions during the data reconstruction.

Electron microscopy, i.e. secondary electron microscopy (SEM) (e.g. [17, 16, 18]), transmission electron microscopy (TEM) (e.g. [7, 15, 20, 21]) or any derivatives thereof, has emerged as a common method to experimentally obtain the APT tip shape. Particularly TEM (tomography) is currently considered as a prospective solution to document the (3D) tip shape in serial or simultaneous TEM-APT measurements with projects initiated to develop combined APT/TEM instruments [3, 22, 23]. However, revealing the 3-dimensional shape of the tip requires TEM-imaging at multiple angles $[24,25,26]$ whereas the (high) electron beam dose associated with this might result in potential carbon build-up and damage accumulation [3] which might compromise subsequent atom probe measurements. Opposed to that, atomic force microscopy (AFM), which is a method extensively used to determine directly and quantitatively complex topographical shapes down to even atomic resolution [27], appears to be a very promising alternative [3] to image the 3-dimensional shape of an atom probe tip at high resolution. AFM does not suffer from radiation damage, is UHV compatible, normally 
introduces no damage when done at minimal force or in non-contact mode, and is relatively cheap. These characteristics render AFM a very interesting, complementary avenue for (insitu) 3D tip shape imaging. Nevertheless, the feasibility of probing the APT-tip shape with AFM has not been proven yet, the challenge being that two sharp tips need to be aligned to each other.

This paper will demonstrate for the first time that AFM can indeed yield easy and fast access to an atom probe tip shape. An approach solving the tip-to-tip alignment problem is exemplified on a fresh, pre-sharpened silicon atom probe tip exhibiting an apex radius of less than $15 \mathrm{~nm}$. The AFM concept is subsequently applied to two APT tips who have been run in an APT-experiment. The APT experiments are representative for the two main causes for specimen end-form deformations, i.e. (i) non-uniform temperature distribution at the apex (UV-laser induced asymmetrical heating [18]) and (ii) differences in the evaporation field thresholds between atoms in a heterogeneous system. The AFM results indicate in both cases the clear deviation from the ideal hemispherical shape illustrating the need for their actual determination in routine measurements.

\section{Materials and methods}

Laser-assisted (UV) atom probe measurements were performed in a LEAP 5000XR on commercially available single crystalline (001) Sb-doped silicon pre-sharpened microtips ${ }^{\mathrm{TM}}$ (PSM) [28] as well as in a LaWatap on an atom probe tip containing a SiGe fin embedded in $\mathrm{SiO}_{2}$, prepared by standard focused ion-beam milling (FIB) lift-out $[29,30]$ and mounted on a tungsten micro-post.

Measurement conditions for the silicon tip were $500 \mathrm{kHz}$ pulse repetition rate, a base temperature of $50 \mathrm{~K}$ and a UV laser energy of $50 \mathrm{pJ}$, which corresponds to an average of $56 \%$ $\mathrm{Si}^{++}\left(44 \% \mathrm{Si}^{+}\right)$charge state abundancy. Data were acquired at a constant detection rate of 0.02 atoms/pulse.

The SiGe fin was analyzed with $100 \mathrm{kHz}$ pulse repetition rate, $80 \mathrm{~K}$ base temperature and a UV laser energy of $50 \mathrm{~nJ}$, which corresponds to an average of $47 \% \mathrm{Si}^{++}$and $35 \% \mathrm{Ge}^{+}$charge state abundancy. Data were acquired at a constant detection rate of 0.001 atoms/pulse. More details on sample preparation and measurement condition can be found in reference [4]. APT data reconstruction was performed using Tap3D (Cameca) applying the cone shape algorithm with a $10^{\circ}$ cone angel and a $80 \AA$ initial curvature radius.

The AFM analysis was performed on a Bruker Dimension Icon operated in tapping mode AFM (amplitude modulation [31]) using the Olympus OMCL-AC160TS silicon probes with a specified apex radius of $7 \mathrm{~nm}$, a spring constant of $26 \mathrm{~N} / \mathrm{m}$ and a typical resonance frequency around $300 \mathrm{kHz}$. We typically operate in a high amplitude regime $(150-300 \mathrm{mV}, 10-20 \mathrm{~nm})$ to minimize the force exerted on the sample both during alignment and measurement. 

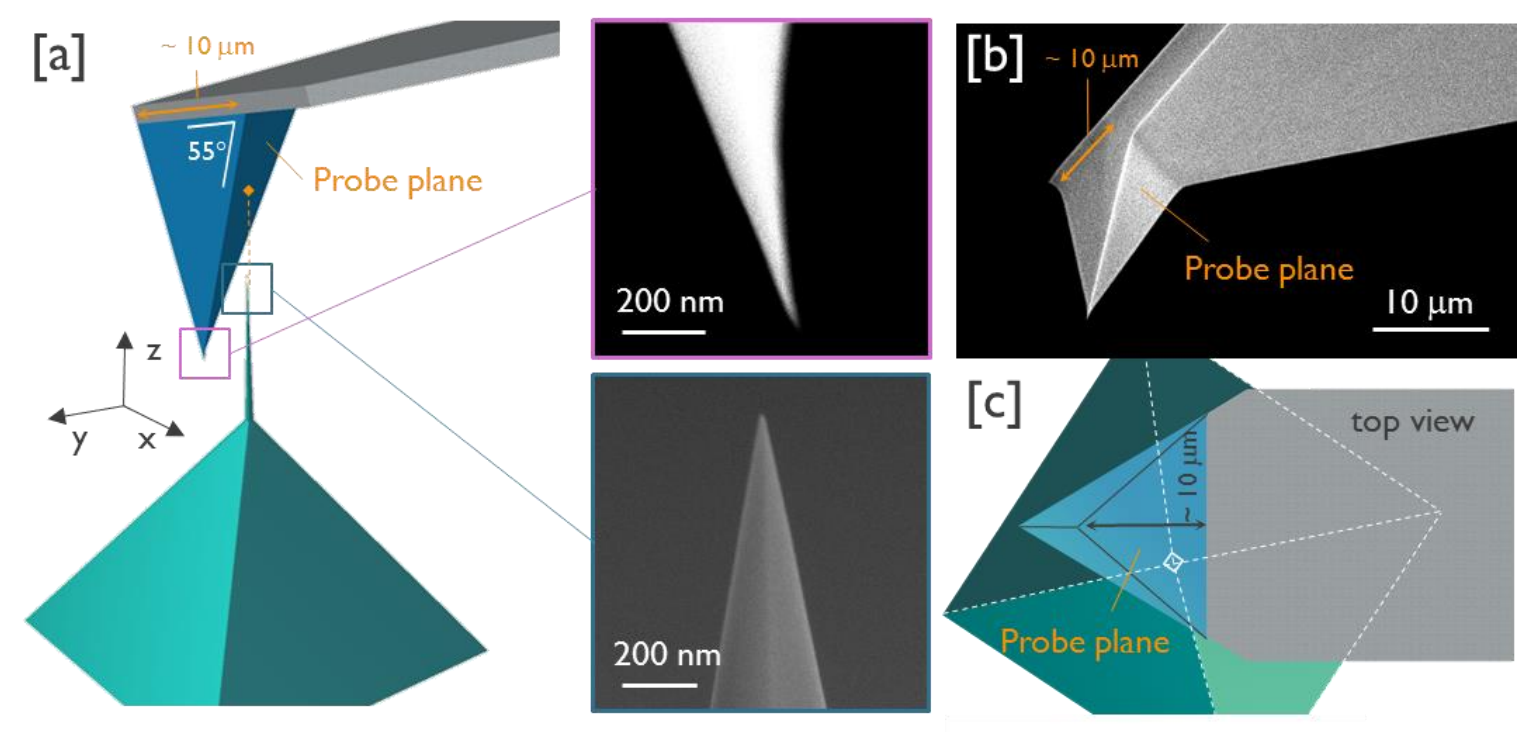

Figure 1: Concept of the tip-to-tip approach [a] with the insets showing SEM pictures of the AFM and PSM tip apices. In [b] the probe plane used for the approach is indicated on an SEM image. The top view schematic in [c] depicts the view from the AFM optics after aligning the APT tip with the AFM probe.

The key challenge of measuring the atom probe tip with an AFM probe is the alignment of the two sharp tips on top of each other. To achieve this, we have exploited the tetrahedral shape of the AFM probe. As depicted in Fig. 1, the triangular plane running into the cantilever has a

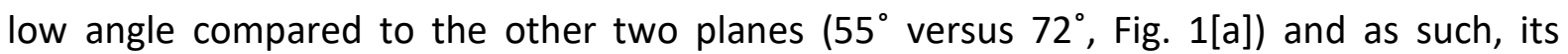
projection covers a large part of the cantilever apex ( $10 \mu \mathrm{m}$, Fig. 1[b]). This enables us to optically position this plane above the atom probe tip (Fig. 1[c]) and to land the AFM probe onto the atom probe tip, as indicated by the diamond symbol in Fig. 1[a]). As the probe plane approaches the APT tip, the resonance frequency increases and the amplitude decreases. The feedback of the AFM system aims to keep the amplitude constant and will thus stop the approach when it senses the APT specimen. Once the AFM probe is in contact with the APT tip, the feedback system of the AFM tool further ensures that we can move the AFM tip freely in the $x$ - and $y$-direction without damaging the APT specimen by adjusting the height of the AFM tip accordingly. In order to align the two tips on top of each other, the AFM tip is moved in the $x$-direction as depicted in Fig. 2 . The APT tip contact location will move from point $A$ to point $B$ and during this movement the AFM tip will initially move up, until the contact location reaches the edge of the AFM probe after which the AFM tip will lower again (see Fig. 2 inset). Upon reaching the edge, the AFM tip is shifted in the y-direction $(100-200 \mathrm{~nm})$ to position the contact location back onto the AFM probe plane (point C). By repeating this procedure, we essentially move along the probe edge to ultimately reach the AFM tip apex (point E). Note that the final movement is characterized by a much steeper height increase as the AFM tip apex is sharpened to yield a higher aspect ratio. 


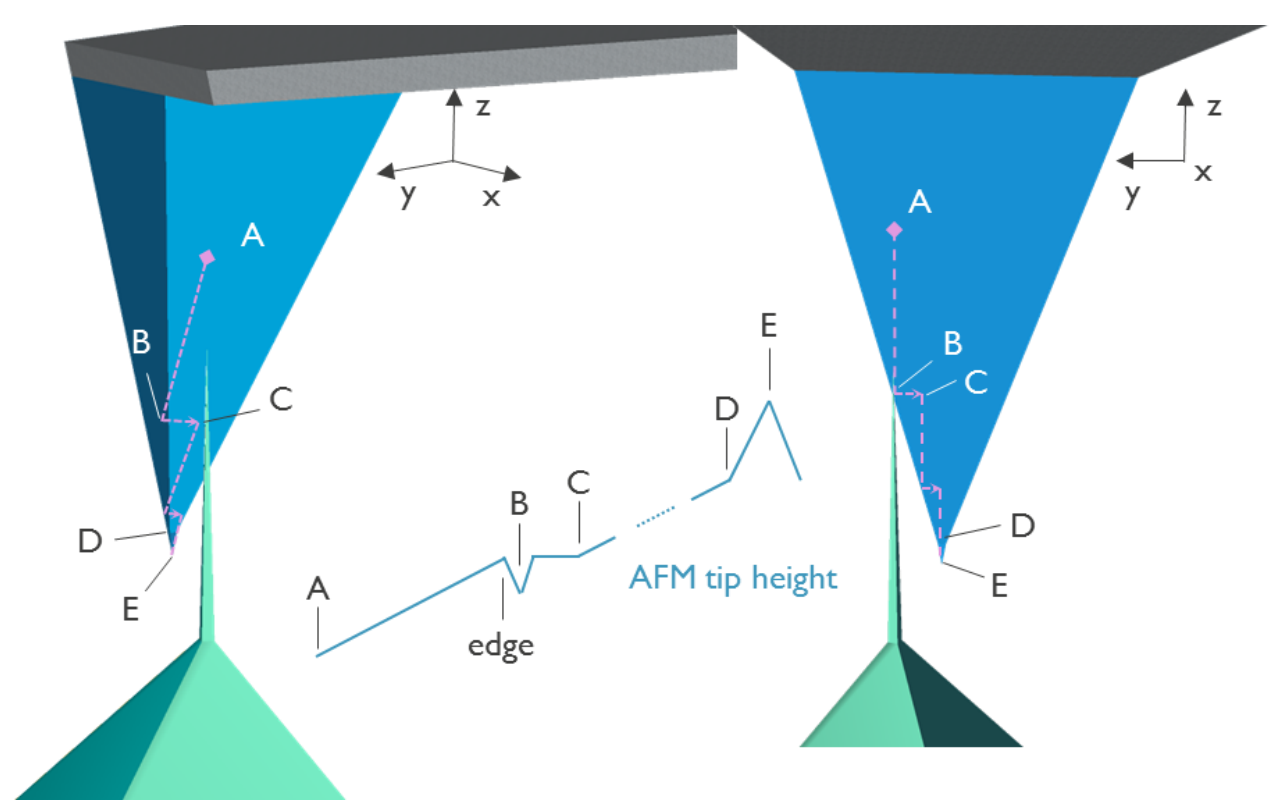

Figure 2: Schematic representation of the tip on tip alignment procedure starting from the initial contact location A after landing. The inset in the middle schematically shows the AFM tip height along the path of the contact location indicated by the dashed line.

\section{Results and Discussion}

Our first target is a fresh, pre-sharpened silicon atom probe tip (PSM) with an apex radius typically less than $15 \mathrm{~nm}$. Never exposed to a high standing voltage or laser pulse, this tip exihibits a point sharp apex and very high aspect ratio, and hence represents the ultimate challenge for the AFM analysis. The insets of Fig. 1[a] display the SEM images of both the AFM probe and the PSM and they highlight the true challenge of measuring a $\sim 15 \mathrm{~nm}$ sharp object with a $7 \mathrm{~nm}$ AFM probe. Nonetheless, using the afore described method we were able to successfully position the AFM probe on top of the PSM and to subsequently image the sharp atom probe tip and part of its shank as evidenced in Fig. 3. Figure 3[a] shows the 3D representation of the entire AFM measurement whereby it is important to note that due to the high aspect ratio of the PSM, the $z$-axis is compressed compared to the $x$ - and $y$-scale. In Fig. 3[b] a zoom of the apex is presented with an uncompressed $z$-scale reflecting the true geometry of the measurement. These data clearly demonstrate that we have succeeded in positioning the two sharp probes on top of each other and in imaging the PSM. 

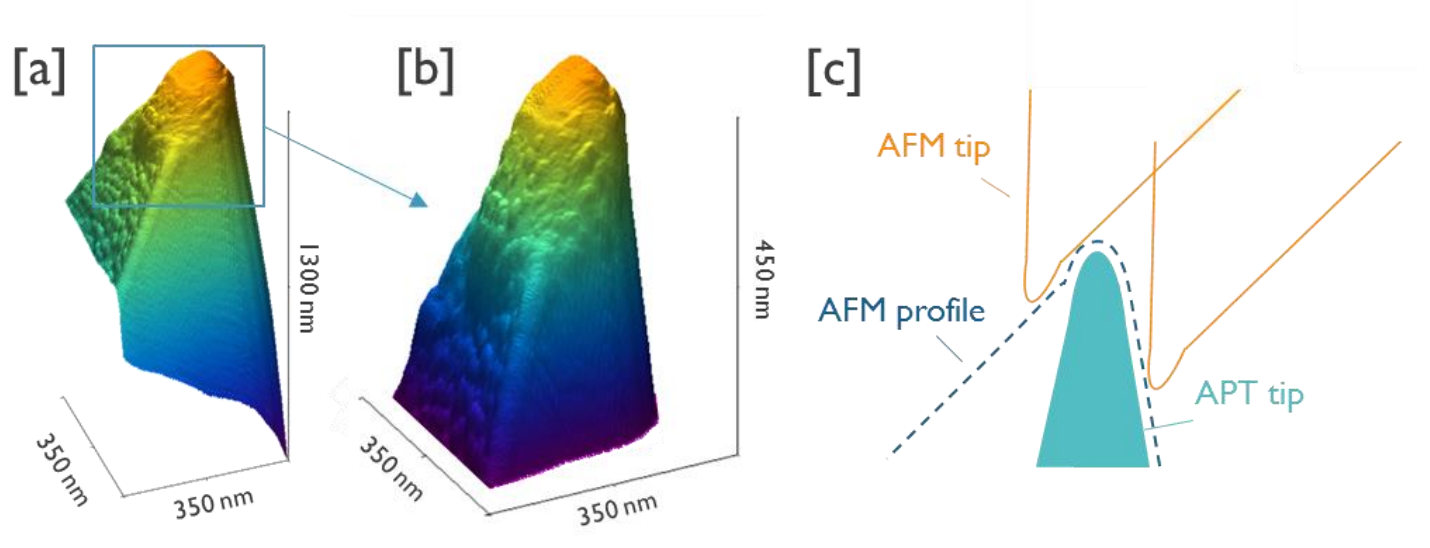

Figure 3: Fresh silicon micro post tip (PSM) as measured by AFM. Apex and shank are detected [a], while a zoom-in on the apex [b] reveals its small radius. [c] Tip artefacts due to the geometry of the AFM probe.

However, by comparing the 3D AFM image with the SEM picture (Fig. 1[a]), it is clear that the overall AFM shape deviates from the expected needle like shape, even though SEM before and after AFM analysis revealed that none of the tips were damaged. A clear distinct triangular plane can be observed where one would expect a very sharp shank. This plane corresponds to the probe plane indicated in Fig. 1 and it demonstrates a well-known limitation of the AFM concept i.e. tip convolution, which is especially observable when the object (i.e. atom probe tip) and probe (i.e. AFM tip) are of comparable sharpness [40]. As long as the relative aspect ratio (and apex radius) of the AFM tip is higher (lower) than that of the atom probe tip, the AFM tip will remain the imaging probe. However, when violated, probe and object will switch roles and the atom probe tip will effectively be probing the AFM tip, as exemplified in Fig. 3[c]. In this scenario, the final image is thus a convolution of both the shape of the AFM and the atom probe tip, as evidenced at the base of the three dimensional AFM image, in which the full tetrahedral shape of the AFM probe can be distinguished. For the sharp PSM, not only the shank but also the measured apex shape will be impacted and its dimensions will be overestimated (with the AFM tip radius, $\sim 7 \mathrm{~nm}$ ) because of the finite size of the AFM probe. This is expected to be less critical when imaging "real" atom probe tips (e.g. during/after APT analysis) for which the average apex diameter will be generally larger than that of the AFM probe. The extent of the convolution thus depends critically on the AFM probe size and shape relative to the specimen and is especially visible on steep edges. These results emphasize that a correct interpretation requires careful data analysis and probe deconvolution possible through the use of probe qualification standards [32, 33, 34, 35, 36, $37,38,39,40]$. This probe deconvolution and the nanoscale interaction of the probe with the APT specimen are the subject of further investigations.

Having shown successful tip-to-tip alignment and AFM measurements on a very sharp atom probe tip, we continue demonstrating the capabilities of AFM to reveal apex shape perturbations such as those stemming from UV laser illumination. As reported previously [18], the asymmetrical tip shape formation is driven by the low penetration depth ( $20 \mathrm{~nm}$ [18]) of UV laser light into $\mathrm{Si}$ and the resulting non-uniform temperature distribution at the apex. This leads locally to a higher evaporation probability and local flattening of the tip, until the shape 
modification induces a sufficient reduction in electric field compensating the local temperature differences. As such the tip will develop a larger radius on the laser-illuminated (higher temperature) side as can be seen by SEM in Ref [18] and Figure 4[a]. Our AFM measurements perfectly capture this asymmetry of the apex (Fig. 4[b]) and demonstrate its 3D-elliptical end-form, which had been observed previously with SEM [9]. Visually a very good agreement is achieved when overlaying the SEM image in Fig. 4[a] with the projected shape of the 3D AFM data (Fig. 4[b]) along a similar viewing angle. The APT tip diameter at $100 \mathrm{~nm}$ below the top is approximately $246 \mathrm{~nm}$ as measured by SEM, while in AFM the diameter varies between 219 and $264 \mathrm{~nm}$ (Fig. 4[d]). This is a good agreement keeping in mind that it is difficult to quantitatively compare length scales from the projected intensity data by SEM with 3D AFM data. Moreover, the projection angle in the SEM-image is not accurately known. It is very encouraging that even the shank of the tip more than $200 \mathrm{~nm}$ away from the apex is nicely represented by the AFM data whereby of course one side of the shank is hidden because of the aforementioned AFM-tip convolution artefact (backside in Fig. 4[b]). However, this can easily be overcome by rotating the atom probe tip to measure both sides of the shank with optimal resolution. Comparing the full 3D information obtained by AFM with the SEM image (Fig. 4[a]), it becomes clear that we must be cautious with the use of "nominal" values for tip radii extracted from $2 \mathrm{D}$ images using circle fit routines.
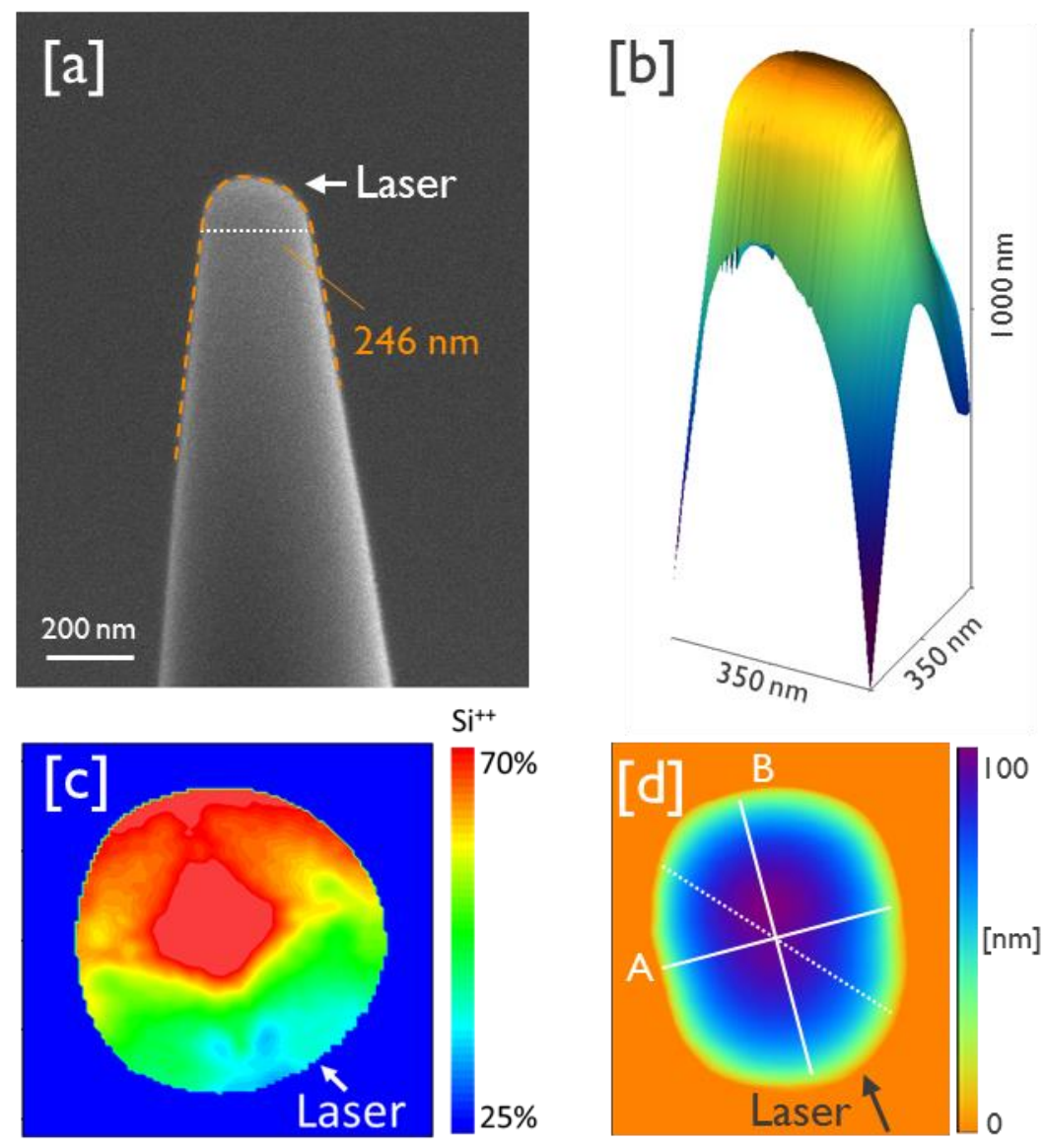

Figure 4: [a] SEM image taken after APT analysis. The dashed line represents the projected shape of the 3D AFM image [b] while the dotted line is a measure taken at approximately $100 \mathrm{~nm}$ from the top same as shown in [d]. [c] Spatially resolved $\mathrm{Si}^{++}$concentration over the tip surface showing the difference in field strength 
between the laser (low field) and shadow side (high field) originating from the shape asymmetry (voxel size $\left.1 \times 1 \times 50 \mathrm{~nm}^{3}\right)$. [d] AFM 2D image of the top $100 \mathrm{~nm}$ of the atom probe tip $(A=219 \mathrm{~nm}, B=264 \mathrm{~nm}$, dotted line $=253 \mathrm{~nm}$ ). The dotted line represents the viewing direction as seen in [a].

The existence of such an asymmetric tip shape is also supported in the APT-data by observing the variation of the $\mathrm{Si}^{++}$concentration over the tip surface (Fig. 4[c]). Indeed due to the fielddependence of the post-ionization process (Kingham curves, [41]) a higher $\mathrm{Si}^{++}$concentration will indicate a higher electric field, implying that the spatial distribution of the $\mathrm{Si}^{++}$ concentration reflects the local electric field distribution in the vicinity of the specimen surface. As the electric field $F$ is controlled by the tip radius $R, \mathrm{~F} \sim \mathrm{V} / \mathrm{kR}[1]$, with $\mathrm{V}$ the applied voltage and $k$ a geometric field constant, its variation will reflect differences in tip radius. In other words, the lower abundancy $\left(\sim 36 \% \mathrm{Si}^{++}\right)$of the double charged silicon atoms on the laser side reflects a lower electric field ( $18 \mathrm{~V} / \mathrm{nm}[41])$ and thus the larger radius as opposed to the shadow side which displays a higher abundancy $\left(\sim 75 \% \mathrm{Si}^{++}\right)$, higher field $(\sim 20 \mathrm{~V} / \mathrm{nm}$ [41]) and thus smaller radius. It is clear that probing the 3D shape of the specimen (as demonstrated here with AFM) offers now an avenue for a more direct and quantitative correlation between the apex end-form, the charge state distribution and electric field. This allows for a clear differentiation between the local and global tip radii (mean-curvature) and their respective electric field strength/distribution.

When dealing with complex hetergonuous samples, one strives to resolve more complex shape variations even over small length scales as the tip shapes will be controlled by the presence of both high and low field materials (e.g. precipitates, multilayer, 3D structures). An example is shown in Fig. 5, which presents the tip shape after partial atom probe analysis of an $\sim 60 \mathrm{~nm}$ wide $\mathrm{SiGe}$ fin embedded in $\mathrm{SiO}_{2}$ (Fig. 5[a]). Here, variations in the apex shape occur over a length scale of less than $100 \mathrm{~nm}$ and are mainly driven by the high evaporation field difference between $\mathrm{SiO}_{2}$ and $\mathrm{SiGe}$, as explained in great detail in [4]. The lower evaporation probability of $\mathrm{SiO}_{2}$ leads to its slower removal versus the SiGe region which is evaporated preferentially. The resulting tip shape, as measured with SEM (Fig. 5[a]) and as predicted with simulations (Fig. 5[d]), deviates substantially from the hemispherical shape. Note that depending on the viewing angle (cfr. the two projections from the simulations) its shape varies from convex to concave calling for a complete 3D-characterization as performed with our AFM-approach (Fig. 5[b]). Such a non-hemispherical tip shape results in a non-uniform magnification over the tip surface. In the reconstructed data (Fig. 5[c]) this appears as distorted shapes, faulty dimensions and/or wrong layer sequences [4, 10, 14, 42]. For example, the actual fin width (Fig. 5[a] and [4]) is artificially compressed in the reconstructed APT data (Fig. 5[c]), which becomes more prominent with depth (z-direction). The origin of this compression lies in the convergence of the ion trajectories above the SiGe region (lower magnification) as a result of the peculiar tip shape in this region (Fig. 5[d] and [4]). The extent of the compression is determined by the ratio of the $\mathrm{SiGe}$ to $\mathrm{SiO}_{2}$ width [4], which due to the cone angle of the APT tip and the tappered side walls of the fin, decreases in $z$ direction (Fig. $5[a])$ and hence increases the compression with depth. A similar artefact can be discerned on the left side of the APT volume (Fig. 5[c]) where the $\mathrm{SiO}_{2}$ layer (shown in red/green) is placed on top of the SiGe layer (shown in red/blue) as opposed to the right side and the actual layer structure.

The AFM measurement on the atom probe tip (Fig. $5[\mathrm{~b}]$ ) resolves the fine structure of the apex, and a measure of the top region ( $66 \mathrm{~nm}$ wide) correlates well with the width of the 
fin. However, we emphasize that for such a complex structure an accurate and quantitative comparison between the SEM and AFM image poses various challenges. Firstly, the SEM image is a 2D intensity projection of a 3D object and image contrast formation might be affected by charging (as is the case in the $\mathrm{SiO}_{2}$ ) and topography. Moreover, the SEM (in our study) does not achieve high enough resolution to resolve the fine features at the apex. On the other hand, AFM measurements partially suffer from tip artefacts at the shank region, which require deconvolution routines to be applied. This can for instance be observed in the 3D AFM image in Fig. 5[b]. The left hand side is convoluted with the probe plane as detailed above, hiding the real shank, whereas the right hand side is imaged with the sharpest probe edge and thus reflects the real morphology of the shank. This also explains why the right hand side of the SEM and AFM image visually agree very well, but much less at the left hand side. As discussed previously these limitations can be overcome by rotating the sample during the AFM-analysis or using a probe with a higher aspect ratio. Tip deconvolution is a standard approach in AFM-based topography measurements and appropriate software algorithms are abundantly available $[32,33,34,35,36,37,38,39,40]$.

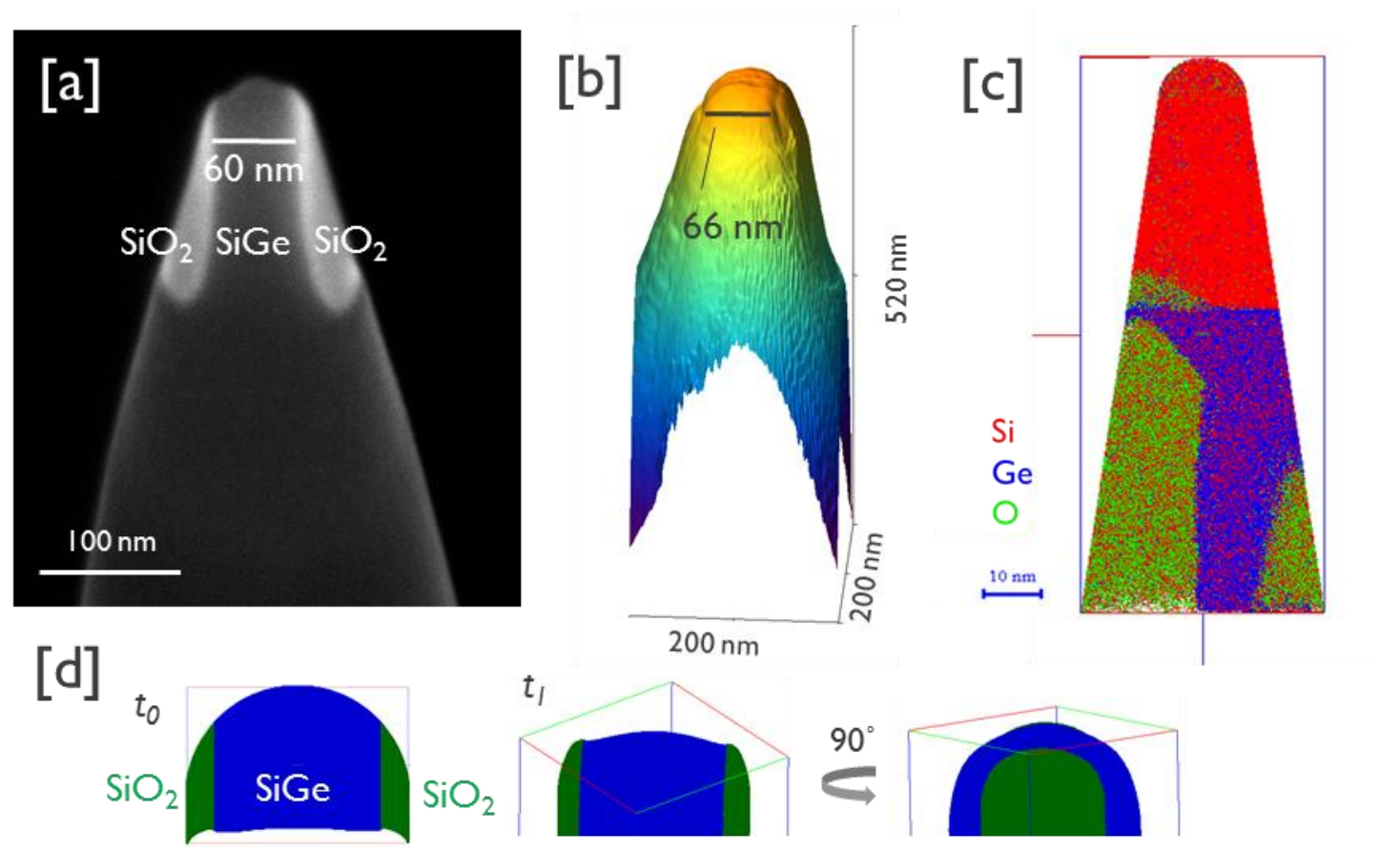

Figure 5: [a] SEM image taken after APT analysis of SiGe fin ( $60 \mathrm{~nm}$ ) embedded between $\mathrm{SiO}_{2}$. [b] 3D AFM image of the fin in [a]. [c] Reconstructed APT data from the analysed region of the same atom probe tip with strong shape deviations arising from the non-hemispherical tip shape that develops during field evaporation as verified by simulations $[d]$.

\section{Conclusion}

We have demonstrated in this work the power of AFM-based measurements to quantitatively assess the 3D shape of an atom probe tip, including its apex and shank region. Tip shape abberrations from an hemispherical end-form can be resolved even at small length scales. The results demonstrate that laser tip interaction effects as well as differences in evaporation fields between constituents of complex systems lead to severe distortions from the ideal 
hemispherical tip shape. In order to reach an improved accuracy with APT analysis, a methodology needs to be adopted whereby the tip shape is included in the ion optical calculations. Since the tip shape dynamics depend on the actual structure under investigation and can even vary during the APT analysis, implementation of a in-situ tip shape monitoring will become a cornerstone towards improved analysis. AFM offers in this respect an effective avenue.

\section{Acknowledgment}

We gratefully acknowledge the funding for this work through the European Metrology Programme for Innovation and Research (EMPIR) Project 3DMetChemIT. The EMPIR initiative is co-funded by the European Union's Horizon 2020 research and innovation programme and the EMPIR Participating States. The authors also acknowledge the financial support by FWOHercules through project ZW13_09.

\section{References}

[1] M. K. Miller and R. G. Forbes, "Atom-Probe Tomography: The Local Electrode Atom Probe," Materials Characterization, vol. 60, no. 6, pp. 461 - 469, 2009.

[2] T. F. Kelly and D. J. Larson, "Atom Probe Tomography 2012," Annu. Rev. Mater. Res., vol. 42, pp. 1-31, 2012.

[3] T. F. Kelly, M. K. Miller, K. Rajan, and S. P. Ringer, "Atomic-scale tomography: A 2020 vision," Microscopy and Microanalysis, vol. 19, pp. 652-664, 62013.

[4] D. Melkonyan, C. Fleischmann, L. Arnoldi, J. Demeulemeester, A. Kumar, J. Bogdanowicz, F. Vurpillot, and W. Vandervorst, "Atom probe tomography analysis of SiGe fins embedded in SiO2: Facts and artefacts," Ultramicroscopy, vol. 179, pp. 100 - 107, 2017. [5] M. Miller and M. Hetherington, "Local magnification effects in the atom probe," Surface Science, vol. 246, no. 1, pp. $442-449,1991$.

[6] S. T. Loi, B. Gault, S. P. Ringer, D. J. Larson, and B. P. Geiser, "Electrostatic simulations of a local electrode atom probe: The dependence of tomographic reconstruction parameters on specimen and microscope geometry," Ultramicroscopy, vol. 132, pp. 107 - 113, 2013.

[7] F. Vurpillot, W. Lefebvre, J. Cairney, C. Oberdorfer, B. Geiser, and K. Rajan, "Advanced volume reconstruction and data mining methods in atom probe tomography," MRS Bulletin, vol. 41, pp. 46-52, 12016.

[8] F. Vurpillot, A. Gaillard, G. D. Costa, and B. Deconihout, "A model to predict image formation in atom probe tomography," Ultramicroscopy, vol. 132, pp. 152 - 157, 2012.

[9] B. Gault, D. Haley, F. de Geuser, M. Moody, E. Marquis, D. Larson, and B. Geiser, "Advances in the reconstruction of atom probe tomography data," Ultramicroscopy, vol. 111, no. 6, pp. 448 - 457, 2011, special Issue: 52nd International Field Emission Symposium.

[10] A. Grenier, S. Duguay, J. Barnes, R. Serra, G. Haberfehlner, D. Cooper, F. Bertin, S. Barraud, G. Audoit, L. Arnoldi, E. Cadel, A. Chabli, and F. Vurpillot, "3D analysis of advanced nano-devices using electron and atom probe tomography," Ultramicroscopy, vol. 136, no. 0, pp. 185 - 192, 2014. [Online]. Available: http://www.sciencedirect.com/science/article/pii/S0304399113002763 
[11] F. Vurpillot, A. Bostel, and D. Blavette, "Trajectory overlaps and local magnification in three-dimensional atom probe," Applied Physics Letters, vol. 76, no. 21, pp. 3127-3129, 2000. [Online]. Available: http://dx.doi.org/10.1063/1.126545

[12] D. Larson, T. Prosa, B. Geiser, and W. Egelhoff, "Effect of analysis direction on the measurement of interfacial mixing in thin metal layers with atom probe tomography," Ultramicroscopy, vol. 111, no. 6, pp. 506 - 511, 2011, special Issue: 52nd International Field Emission Symposium. [Online]. Available: http://www.sciencedirect.com/science/article/pii/S0304399110003529

[13] D. Larson, B. Geiser, T. Prosa, and T. Kelly, "On the use of simulated field-evaporated specimen apex shapes in atom probe tomography data reconstruction," Microscopy and Microanalysis, vol.18, no. 5, pp. 953-963, 2012. [Online]. Available: http://www.ncbi.nlm.nih.gov/pubmed/23058657

[14] A. Grenier, S. Duguay, J. P. Barnes, R. Serra, N. Rolland, G. Audoit, P. Morin, P. Gouraud, D. Cooper, D. Blavette, and F. Vurpillot, "Three dimensional imaging and analysis of a single nano-device at the ultimate scale using correlative microscopy techniques," Applied Physics Letters, vol. 106, no. 21, 2015. [Online]. Available: http://scitation.aip.org/content/aip/journal/apl/106/21/10.1063/1.4921352

[15] G. Sha, A. Cerezo, and G. D. W. Smith, "Field evaporation behavior during irradiation with picosecond laser pulses," Applied Physics Letters, vol. 92, no. 4, p. 043503, 2008. [Online]. Available: https://doi.org/10.1063/1.2837626

[16] S. Gerstl, B. Geiser, T. Kelly, and D. Larson, "Evaluation of local radii of atom-probetomography specimens," Microscopy and Microanalysis, vol. 15, no. S2, p. 248-249, 2009.

[17] A. Shariq, S. Mutas, K. Wedderhoff, C. Klein, H. Hortenbach, S. Teichert, P. Kücher, and S. Gerstl, "Investigations of field-evaporated end forms in voltage- and laser-pulsed atom probe tomography," Ultramicroscopy, vol. 109, no. 5, pp. 472 - 479, 2009. [Online]. Available: http://www.sciencedirect.com/science/article/pii/S0304399108002611

[18] S. Koelling, N. Innocenti, A. Schulze, M. Gilbert, A. K. Kambham, and W. Vandervorst, "In-situ observation of non-hemispherical tip shape formation during laser-assisted atom probe tomography," Journal of Applied Physics, vol. 109, no. 104909, 2011.

[19] C.-M. Kwak, Y.-T. Kim, C.-G. Park, and J.-B. Seol, "Understanding of Capping Effects on the Tip Shape Evolution and on the Atom Probe Data of Bulk LaAlO3 Using Transmission Electron Microscopy," Microscopy and Microanalysis, vol. 23, no. 2, p. 329-335, 2017.

[20] D. Larson, B. Geiser, T. Prosa, S. Gerstl, D. Reinhard, and T. Kelly, "Improvements in planar feature reconstructions in atom probe tomography," Journal of Microscopy, vol. 243, no. 1, pp. 15-30, 2011. [Online]. Available: http://dx.doi.org/10.1111/j.13652818.2010.03474.x

[21] W. Lefebvre, D. Hernandez-Maldonado, F. Moyon, F. Cuvilly, C. Vaudolon, D. Shinde, and F. Vurpillot, "HAADF-STEM atom counting in atom probe tomography specimens: Towards quantitative correlative microscopy," Ultramicroscopy, vol. 159, pp. 403-412, 2015, 1st International Conference on Atom Probe Tomography \& Microscopy. [Online]. Available: http://www.sciencedirect.com/science/article/pii/S0304399115000261

[22] M. K. Miller and T. Kelly, "The atom tomography (atom) concept," vol. 16, pp. 18561857, 082010.

[23] T. Kelly, M. Miller, K. Rajan, S. Ringer, A. Borisevich, N. Dellby, and O. Krivanek, "Toward atomic-scale tomography: The atom project," Microscopy and Microanalysis, vol. 17, no. S2, p. 708-709, 2011. 
[24] T. C. Petersen and S.P. Ringer, "Electron tomography using a geometric surfacetangent algorithm: Application to atom probe specimen morphology," Journal of Applied Physics, vol. 105, no. 10, p. 103518, 2009. [Online]. Available: http://dx.doi.org/10.1063/1.3129310

[25] T. Petersen and S. Ringer, "An electron tomography algorithm for reconstructing 3D morphology using surface tangents of projected scattering interfaces," Computer Physics Communications, vol. 181, no. 3, pp. 676 - 682, 2010. [Online]. Available: http://www.sciencedirect.com/science/article/pii/S0010465509003440

[26] D. Haley, T. Petersen, S. Ringer, and G. Smith, "Atom probe trajectory mapping using experimental tip shape measurements," Journal of Microscopy, vol. 244, no. 2, pp. 170-180, 2011. [Online]. Available: http://dx.doi.org/10.1111/j.1365-2818.2011.03522.x

[27] F. J. Giessibl, "AFM's path to atomic resolution," Materials Today, vol. 8, no. 5, pp. 32 - 41, 2005. [Online]. Available: http://www.sciencedirect.com/science/article/pii/S1369702105008448

[28] K. Thompson, D. J. Larson, and R. M. Ulfig, "Pre-sharpened and flat-top microtip coupons: a quantitative comparison for atom-probe analysis studies," Microscopy and Microanalysis, vol. 11, no. S02, p. 882-883, 2005.

[29] K. Thompson, D. Lawrence, D. Larson, J. Olson, T. Kelly, and B. Gorman, "In situ sitespecific specimen preparation for atom probe tomography," Ultramicroscopy, vol. 107, pp. 131 - 139, 2007. [Online]. Available: http://www.sciencedirect.com/science/article/pii/S0304399106001203

[30] M. Miller and K. Russell, "Atom probe specimen preparation with a dual beam SEM/FIB miller," Ultramicroscopy, vol. 107, no. 9, pp. 761 - 766, 2007, proceedings of the 50th International Field Emission Symposium. [Online]. Available: http://www.sciencedirect.com/science/article/pii/S030439910700054X

[31] B. Voigtlaender, Scanning Probe Microscopy: Atomic Force Microscopy and Scanning Tunneling Microscopy, ser. NanoScience and Technology. Springer-Verlag Berlin Heidelberg, 2015.

[32] P. Markiewicz and M. C. Goh, "Atomic force microscopy probe tip visualization and improvement of images using a simple deconvolution procedure," Langmuir, vol. 10, no. 1, pp. 5-7, 1994. [Online]. Available: http://dx.doi.org/10.1021/la00013a002

[33] L. Hellemans, K. Waeyaert, F. Hennau, L. Stockman, I. Heyvaert, and C. V. Haesendonck, "Can atomic force microscopy tips be inspected by atomic force microscopy?" Journal of Vacuum Science \& Technology B: Microelectronics and Nanometer Structures Processing, Measurement, and Phenomena, vol. 9, no. 2, pp. 1309-1312, 1991. [Online]. Available: http://avs.scitation.org/doi/abs/10.1116/1.585185

[34] K. L. Westra, A. W. Mitchell, and D.J. Thomson, "Tip artifacts in atomic force microscope imaging of thin film surfaces," Journal of Applied Physics, vol. 74, no. 5, pp. 36083610, 1993. [Online]. Available: https://doi.org/10.1063/1.354498

[35] P. Markiewicz and M. C. Goh, "Atomic force microscope tip deconvolution using calibration arrays," Review of Scientific Instruments, vol. 66, no. 5, pp. 3186-3190, 1995. [Online]. Available: https://doi.org/10.1063/1.1145549

[36] P. Markiewicz and M. C. Goh, "Simulation of atomic force microscope tipsample/sample-tip reconstruction," Journal of Vacuum Science \& Technology B: Microelectronics and Nanometer Structures Processing, Measurement, and Phenomena, vol. 13, no. 3, pp. 1115-1118, 1995. [Online]. Available: http://avs.scitation.org/doi/abs/$10.1116 / 1.587913$ 
[37] J. Villarrubia, "Algorithms for scanned probe microscope image simulation, surface reconstruction, and tip estimation," Journal of Research of the National Institute of Standards and Technology, vol. 102, no. 4, pp. 425-454, 1997.

[38] B. Skårman, L. R. Wallenberg, S. N. Jacobsen, U. Helmersson, and C. Thelander, "Evaluation of intermittent contact mode AFM probes by HREM and using atomically sharp $\mathrm{CeO} 2$ ridges as tip characterizer," Langmuir, vol. 16, no. 15, pp. 6267-6277, 2000. [Online]. Available: http://dx.doi.org/10.1021/la000078t

[39] J. Canet-Ferrer, E. Coronado, A. Forment-Aliaga, and E. Pinilla-Cienfuegos, "Correction of the tip convolution effects in the imaging of nanostructures studied through scanning force microscopy," Nanotechnology, vol. 25, no. 39, p. 395703, 2014. [Online]. Available: http://stacks.iop.org/0957-4484/25/i=39/a=395703

[40] J. Shen, D. Zhang, F.-H. Zhang, and Y. Gan, "AFM tip-sample convolution effects for cylinder protrusions," Applied Surface Science, vol. 422, pp. 482 - 491, 2017. [Online]. Available: http://www.sciencedirect.com/science/article/pii/S0169433217317087

[41] R. Haydock and D. R. Kingham, "Post-Ionization of Field-Evaporated Ions," Phys. Rev. Lett., vol. 44, pp. 1520-1523, Jun 1980. [Online]. Available: https://link.aps.org/doi/10.1103/PhysRevLett.44.1520

[42] D. Melkonyan, C. Fleischmann, A. Veloso, A. Franquet, J. Bogdanowicz, R. Morris, and W. Vandervorst, "Wet-chemical etching of atom probe tips for artefact free analyses of nanoscaled semiconductor structures," Ultramicroscopy, vol. 186, pp. 1 - 8, 2018. [Online]. Available: http://www.sciencedirect.com/science/article/pii/S0304399117303728 